\title{
Técnicas de inhalación en el tratamiento del asma
}

\section{Inhalation techniques in the treatment of asthma}

\section{Rodríguez, E. Celay, I. Larrea, M. Urdánoz, A. Zabalegui, B. Ollo}

\section{RESUMEN}

En el tratamiento farmacológico del asma, la vía más adecuada es la inhalatoria, ya que nos permite alcanzar una mayor concentración de medicamento en el órgano de choque que la medicación sistémica, y además se minimizan los efectos secundarios de esta última.

El uso adecuado de los inhaladores y con ello su eficacia depende de muy diversos factores. Entre los de índole meramente técnica, algunos de ellos son:

- Un adecuado volumen de inhalación.

- El ritmo de inhalación.

- La duración de la apnea inspiratoria tras la administración del medicamento

- La coordinación entre la inspiración del aerosol y la activación del dispositivo, en el caso del MDI

Uno de los motivos de uso incorrecto de los aerosoles MDI es la falta de coordinación entre la activación del dispositivo y la inspiración. Ello ha motivado el diseño de diferentes dispositivos que obvien la necesidad de esta coordinacion como son: las camaras latorias y los dispositivos para inhalar polvo seco.

También se sugiere en la literatura que las causas de estos errores son motivados porque los profesionales sanitarios no conocen o no enseñan adecuadamente el manejo de los dispositivos a los pacientes. A pesar de estar diseñados para facilitar la correcta administe extmistracion, exigen un conocimiento adecuado de la técni-

Palabras clave. Técnicas de inhalación. Educación sanitaria. Inhaladores.

An. sis. sanit. Navar. 2003; 26 (Supl. 2): 139-146.

\section{ABSTRACT}

In the pharmacological treatment of asthma, the most suitable way is by inhalation, since this enables us to achieve a greater concentration of medicine in the affected organ than systemic medication, and besides the latter's secondary effects are minimized.

The suitable use of inhalers and hence their efficacy depends on very different factors. Some of those of a merely technical kind are as follows:

- A suitable volume of inhalation.

- The rhythm of inhalation.

- The length of the inspiratory apnea following administration of the medicine.

- The coordination between the inspiration of the aerosol and activation of the device, in the case of the metered dose inhaler (MDI).

One of the reasons for the incorrect use of MD aerosols is the lack of coordination between activatio of the device and inspiration. This has led to the design of different devices that avoid the need for this coordination, such as: inhalation chambers and devices for inhaling dry dust.

It has also been suggested in the literature that the causes of these mistakes are to be found in the health professionals not knowing, or not suitably teaching the patients how to handle the devices. In spite of being designed to facilitate correct administration, suitable desir use by the patient knowledge of the technique of their use by the patient
is required.

Key words. Inhalation techniques. Health education. Inhalers.
Sección de Alergología. Hospital Virgen del Camino. Pamplona.

\section{Correspondencia:}

Marisa Rodríguez Biempica

Sección de Alergología

Centro de Especialidades Conde Oliveto

Plaza de la Paz s/n

31002 PAMPLONA

Tfno: 948429250

Fax: 948429271 


\section{INTRODUCCIÓN}

$\mathrm{El}$ asma bronquial es una enfermedad inflamatoria crónica de la vía aérea en la que predominan los eosinófilos y que cursa con episodios de obstrucción variable al flujo aéreo, reversibles de forma espontánea o tras medicación y en la que la hiperreactividad bronquial es un rasgo prominente.

Aunque la causa del asma no es del todo conocida, existen unos factores que están implicados en el desarrollo del asma y en la aparición de las crisis ${ }^{1}$ atopia, alergenos domésticos y/o ambientales, sensibilizantes ocupacionales, fármacos, irritantes, infecciones respiratorias, esfuerzo físico solo o tras la ingesta de ciertos alimentos $^{2,3}$, condiciones metereológicas adversas.

Según datos publicados por la OMS, se estima que a nivel mundial la enfermedad podría estar afectando entre 100 y 150 millones de personas y ocasionar una mortalidad directa anual a 2 millones de personas. La morbilidad y mortalidad producidas por el asma han aumentado en las dos últimas décadas en todos los grupos de edad, pero fundamentalmente en niños que viven en medio urbano.

La vía inhalatoria es la más apropiada para que los medicamentos lleguen de forma más eficaz al órgano diana. Nadie cuestiona este aspecto pero tiene el inconveniente de que la técnica de inhalación puede resultar difícil para algunas personas por lo que su prescripción debe acompañarse siempre del adecuado adiestramiento del paciente.

En distintos estudios se ha demostrado que los programas de educación tanto en pacientes asmáticos ${ }^{4,5}$ como en profesionales sanitarios ${ }^{6}$, mejoran la técnica de inhalación, contribuyen a mejorar el cumplimiento y a alcanzar la eficacia terapéutica deseada.

\section{SISTEMAS DE INHALACIÓN}

\section{Aerosoles presurizados}

Los cartuchos presurizados o MDI se definen como sistemas que dependen de la fuerza de un gas comprimido o licuado para expulsar el contenido del envase. Las sustancias terapéuticas pueden estar disueltas, suspendidas o emulsionadas en un propulsor o mezcla de propulsor/disolvente, diseñados para ejercer una acción local o sistémica.

Estos dispositivos disponen de una carcasa, una boquilla y una válvula dosificadora o pulsador que permite la administración uniforme y dosificada del medicamento (Fig. 1).

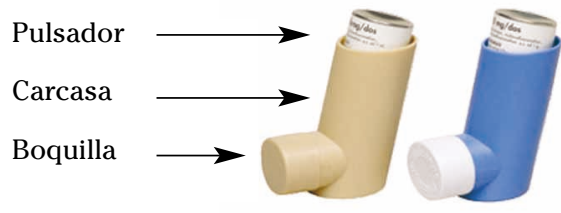

Figura 1. Inhaladores MDI.

El fármaco micronizado está disuelto en diferentes propelentes que actúan como propulsores. Se utilizan los propelentes clorofluorocarbonados (CFCs) porque son fáciles de licuar, no tóxicos, no corrosivos y no inflamables; sin embargo están siendo sustituidos por propelentes menos nocivos para la capa de ozono ( Protocolo de Montreal), según el cual en 1998 se reduciría en un $50 \%$ el uso de $\mathrm{CFCs}^{7}$. Entre estos nuevos propelentes se encuentran los hidrofluoroalkanos (HFA 134 a) que no contienen cloro y en la degradación no producen radicales libres que catalizan la destrucción de la capa de ozono ${ }^{8}$. Este hecho es importante conocerlo para informar a nuestros pacientes de que pueden notar cambios en el sabor y en la presión de salida del medicamento ${ }^{9}$.

Ventajas: son baratos (a corto plazo), transportables y resistentes al agua y más del $70 \%$ de las prescripciones.

Inconvenientes: la técnica de uso es compleja, no adecuado para niños, difícil de monitorizar la dosificación, no respeta el medio ambiente; no es adecuado en situaciones de broncoespasmo intenso y elevado depósito orofaríngeo.

\section{Recomendaciones (Barley C)}

- No usar el inhalador si está por debajo de la temperatura ambiente; la presión 
interna del inhalador es sensible a la temperatura

- La postura correcta del inhalador es en forma de L, para evitar la salida del propelente sin medicamento.

- Postura correcta del paciente (erguido o sentado), para permitir la máxima expansión torácica.

- La cabeza ligeramente inclinada hacia atrás, para formar un canal lo más recto posible para el inhalador.

- La lengua relajada en el suelo de la boca, así produce menos obstrucción al flujo aéreo.

La respiración profunda y lenta, la inhalación lenta reduce el depósito de partículas en las vías respiratorias superiores.

\section{Técnica}

1. Retirar la tapa del inhalador.

2. Agitar el inhalador para conseguir una adecuada dispersión del propelente y del medicamento.

3. Vaciar completamente el pecho de aire.

4. Colocar la boquilla del cartucho en la boca, sujetándola con los dientes y cerrando los labios alrededor.

5. Una vez iniciada la inspiración, pulsar el inhalador una sola vez, y continuar la inhalación lenta y profundamente hasta llenar los pulmones.
6. Retirar el inhalador de la boca y retener el aire inspirado (pausa inspiratoria) durante 10 segundos (facilita el máximo depósito de las partículas del medicamento y reduce la cantidad de dosis inhalada que se exhala).

7. Espirar como en una respiración normal, lentamente y por la nariz (minimiza la cantidad de medicamento depositado en la boca y que luego es tragado)

8. Esperar 1 minuto antes de aplicarse una segunda dosis (para que se recupere la presión de la válvula).

\section{Errores que más se repiten en el MDI:}

No agitar antes el inhalador, no vaciar el aire, pulsar directamente en la boca y después inspirar, dejar de inspirar una vez pulsado el inhalador y hacer las 2 pulsaciones seguidas.

\section{Mantenimiento}

Es necesario tapar el cartucho y guardar en un lugar seco, mantener el inhalador limpio (especialmente en la zona de la boquilla) y lavar una vez por semana la carcasa.

\section{Cámaras de inhalación}

Las cámaras espaciadoras son dispositivos que aumentan la distancia entre el cartucho presurizado y la boca del paciente (Fig. 2). Produce un ralentizamiento en la velocidad de salida del aerosol. Están diseñadas para facilitar la coordinación

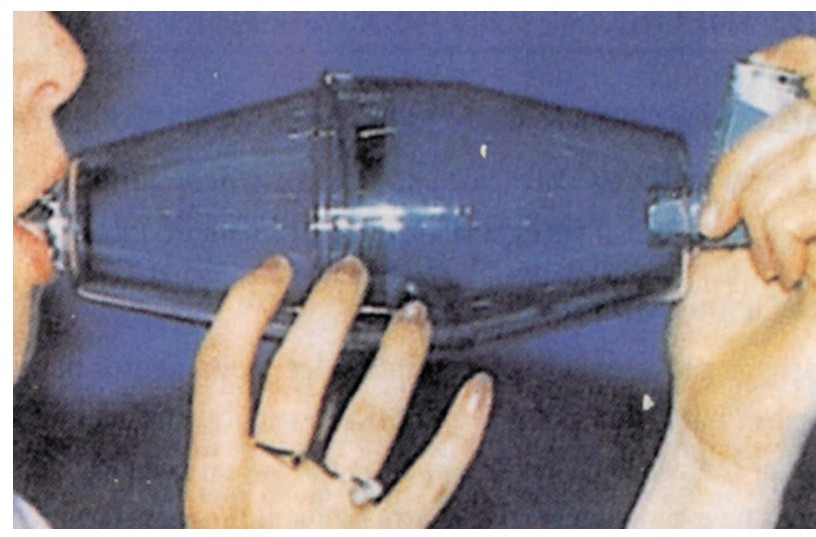

Figura 2. Cámaras espaciadoras. 
del disparo del cartucho y la inspiración del paciente.

Un aspecto que debe considerarse es el número de pulsaciones seguidas en una cámara. En diversos estudios ${ }^{11,12}$ se demuestra que múltiples pulsaciones no consiguen más concentración de fármaco en los pulmones. Así mismo en otras publicaciones se considera que la utilización de cartuchos presurizados con cámaras espaciadoras es la forma más recomendable para la terapia de pacientes con patologías respiratorias ${ }^{13,14}$

Un estudio realizado por Barry y col $^{15}$ concluye que existen diferencias entre la cantidad de fármaco disponible para la inhalación en función del tipo de cámara utilizada.

Ventajas: son baratos (a corto plazo), no es necesaria la inspiración coordinada, es adecuado en situaciones de broncoespasmo intenso, y obstrucción habitual al flujo aéreo, adecuado para niños, depósito orofaríngeo reducido y periférico aumentado y es de elección para administrar dosis elevadas de esteroides inhalados.

Inconvenientes: son menos transportables, difíciles de monitorizar la dosificación, no respetan el medio ambiente, es necesario adiestramiento exigente, disminución intensa de fármaco administrado (si no se sigue la metódica), susceptible a los efectos de la carga estática y el aumento de la biodisponibilidad pulmonar puede incrementar los efectos secundarios.

Las más recomendables son las denominadas de alto volumen, que en el caso de los adultos son de unos $750 \mathrm{ml}$ y en niños hasta \pm 6 años de $150-350 \mathrm{ml}$, en los cuales hasta los 4 años se recomienda el uso de cámara con mascarilla y a partir de esta edad sin ella.

\section{Técnica para cámara sin mascarilla}

Técnica para cámara sin mascarilla para cámaras adultos y cámaras infantiles en niños $>4$ años $^{16}$ :

1. Colocación de pie o sentado.

2. Destapar el inhalador.

3. Colocar en posición correcta (en forma de L).
4. Agitar el inhalador unos segundos.

5. Acoplar el inhalador en el orificio de la cámara en posición vertical. unda.

6. Realizar una espiración lenta y pro-

7. Colocar la boquilla de la cámara en la boca, sujetándola con los dientes y ajustando los labios a la cámara.

8. Efectuar una pulsación del inhalador e inspirar lenta y profundamente.

9. Retirar la cámara de la boca, mantener la inspiración 10 segundos y expulsar lentamente el aire por la nariz.

10. En caso de necesitar una $2^{\mathrm{a}}$ dosis, esperar un minuto para que la válvula recupere la presión.

Recomendaciones en niños

Enseñar al niño a coger el aire llenando bien el pecho sin elevar los hombros, tener una postura correcta de pie o sentado (si el niño colabora) y si es lactante o bebé o no colabora, coger al niño y sujetarlo bien (Fig. 3).

\section{Técnica para cámara con \\ mascarilla (niños $\leq 4$ años)}

Es común a las otras cámaras. Las actuaciones específicas de esta cámara van referidas a los siguientes puntos:

1. Colocar la mascarilla sobre nariz y boca del niño.

2. Mantener la cámara ligeramente inclinada hacia arriba, y seguir en esta

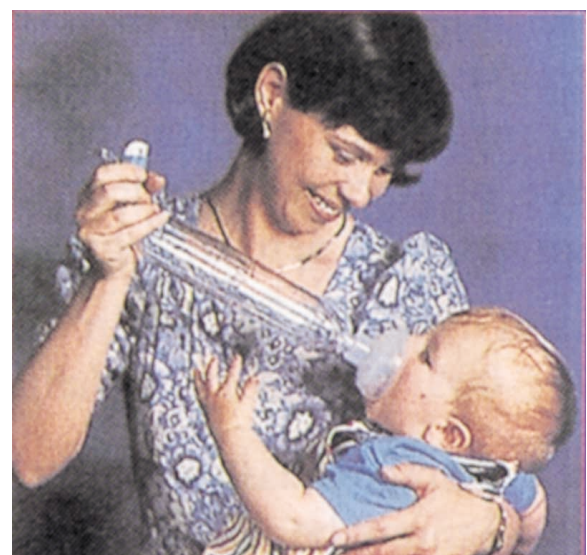

Figura 3. Recomendaciones en niños. 
posición hasta que el niño haya respirado de 5 a 10 veces (15 segundos).

3. Apartar la mascarilla de la cara del niño.

4. Lavarle la cara después de utilizar la cámara.

5. Retirar el inhalador de la cámara y colocar la tapa de la boquilla en el inhalador.

6. Practicar de vez en cuando con el niño, para que se acostumbre al uso de la cámara o para que no se le olvide si la usa esporádicamente.

\section{Mantenimiento}

Se debe realizar una vez por semana: desmontar, lavar con agua y jabón, secar bien, volver a montar, comprobar el buen funcionamiento de la(s) válvula(s).

\section{Errores}

Son errores: introducir más de una dosis en la cámara antes de inhalar y realizar inspiraciones rápidas y superficiales.

\section{Inhaladores de polvo seco}

Estos sistemas contienen el principio activo en forma de polvo. Son dispositivos
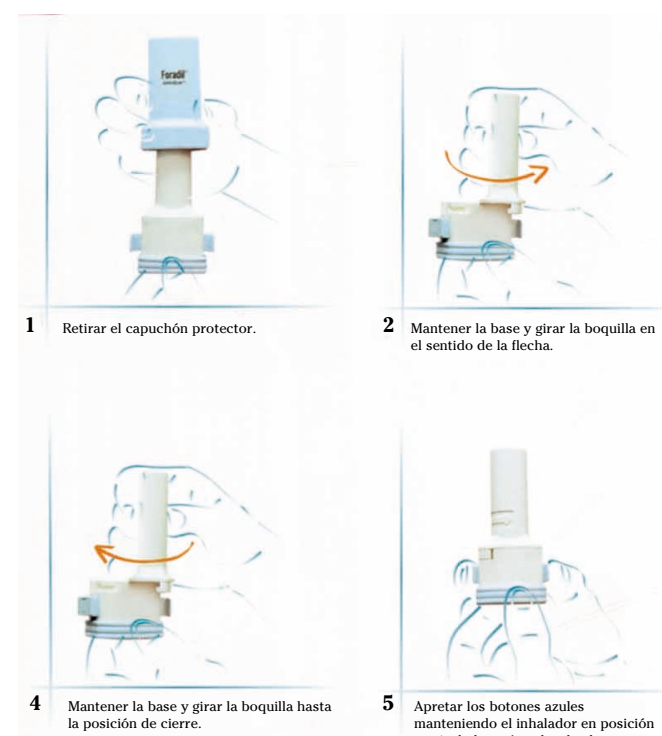

5

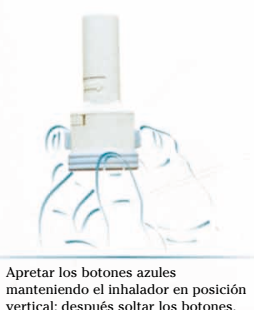

accionados por la inspiración. Aparecen como alternativa a los cartuchos presurizados, con la intención de solucionar los problemas de coordinación entre inhalación y activación del dispositivo.

Ventajas: son fáciles de usar, respetan el medio ambiente, incrementan el depósito pulmonar (central), disminuyen efectos secundarios locales y sistémicos y son adecuados para niños a partir de 6 años.

Inconvenientes: encarece el tratamiento, la eficacia en condiciones extremas es mala, activados por el flujo inspiratorio (difícil de conseguir en niños y pacientes con $\mathrm{EPOC}^{17,18}$ y se necesita un flujo aéreo inspiratorio mínimo de 30-60 litros/minuto.

En función del número de dosis del fármaco que proporcionan, se clasifican en: inhaladores de polvo seco unidosis e inhaladores de polvo multidosis.

\section{Inhaladores de polvo seco unidosis}

Son cápsulas u óvalos con una sola dosis de medicamento que deben perforarse para su inhalación. Cada uno lleva su dispositivo específico (Fig. 4).

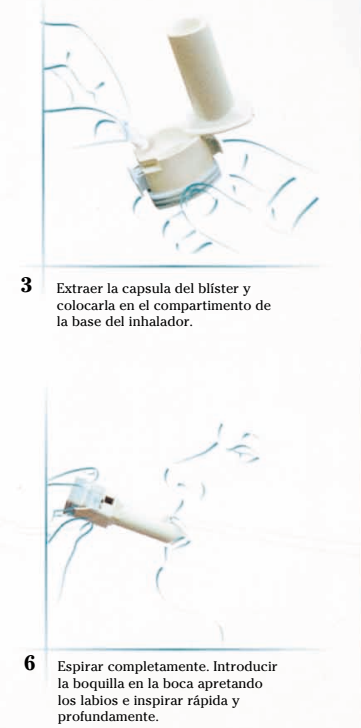

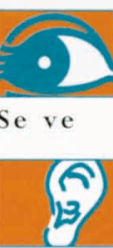

Se oye

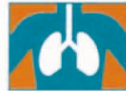

Se nota

Figura 4. Manejo de los inhaladores de polvo seco unidosis 


\section{Técnica}

1. Abrir el inhalador.

2. Colocar la cápsula en el orificio previsto para ello y cerrar el inhalador.

3. Apretar el pulsador hasta el fondo (se rompe la cápsula y la sustancia activa está preparada para ser inhalada).

4. Ponerse de pie o sentado.

5. Expulsar el aire por la boca, manteniendo el inhalador apartado de la misma.

6. Colocar la boquilla entre los dientes y cerrar los labios alrededor, dejando la lengua apoyada en el suelo de la boca.

7. Aspirar enérgica y profundamente, incluso si es preciso 2 veces, hasta lograr vaciar el contenido de la cápsula.

8. Retener el aire 10 segundos.

9. Espirar lentamente por la nariz.

10. Abrir el dispositivo, retirar la cápsula vacía y cerrar el inhalador.

\section{Mantenimiento}

Guardarlo en lugar seco y limpiar el inhalador una vez por semana con agua y jabón, aclarar y secar bien para que no se quede adherido el polvo en la boquilla o filtro.

\section{Inhaladores de polvo seco \\ multidosis}

Existen varios sistemas comercializados de polvo seco en multidosis: Accuha- ler® (sustituye al Diskhaler® ), Clickhaler ${ }^{\circledR}$, Easihaler ${ }^{\circledR}$ y Turbuhaler ${ }^{\circledR}$.

Los más utilizados son: Accuhaler y Turbuhaler.

\section{Accuhaler ${ }^{\circledR}$}

Dispositivo que contiene 60 dosis de medicamento, cubiertas y protegidas individualmente en blisters de aluminio termosellados. Está provisto de un contador de dosis, para facilitar al paciente el número de dosis que le quedan disponibles (Fig. 5)

Contiene como excipiente lactosa, por lo que el paciente percibe la inhalación del fármaco.

Comparados los dispositivos Accuhaler y Turbuhaler en pacientes con EPOC avanzada, se ha comprobado que con el dispositivo Accuhaler se consigue una mayor exactitud / reproductibilidad de dosis, independiente del flujo inspiratorio del paciente ${ }^{18}$.

Técnica: colocarse de pie o sentado, abrir el dispositivo hasta donde haga tope, deslizar la palanca hasta oír un sonido "clic", el dispositivo está preparado para su uso. Espirar profundamente manteniendo el dispositivo alejado de la boca, la cabeza debe estar inclinada ligeramente hacia atrás, colocar la boquilla en la boca, inspirar progresiva e intensamente a través del dispositivo, sacar el dispositivo de la boca y mantener 10 segundos la inspira-

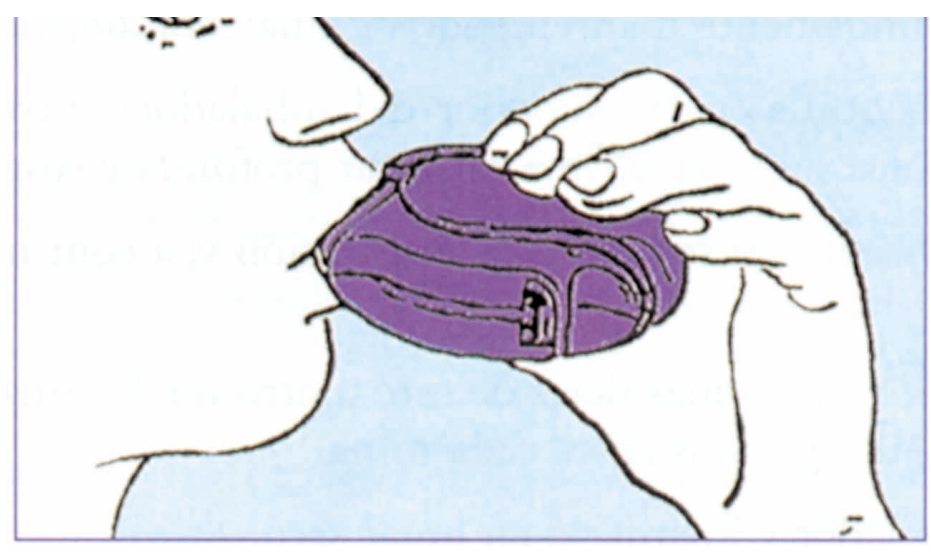

Figura 5. Sistema Accuhaler. 
ción facilitando así el máximo depósito de las partículas del medicamento y reduciendo la cantidad de dosis inhalada que se exhala, espirar lentamente por la nariz y esperar un mínimo de 30 segundos si se debe repetir una segunda dosis del mismo u otro medicamento.

Mantenimiento: limpiar la boquilla con frecuencia para evitar depósitos del producto y guardar en lugar seco.

Error: no bajar la palanca.

\section{Turbuhaler}

Es un dispositivo que contiene 100-200 dosis de fármaco micronizado en un depósito. Está provisto de un disco giratorio dosificador que al girar deposita la dosis del fármaco para la inhalación. Dispone de un indicador que avisa al paciente cuando quedan 20 dosis, y en algunas de sus presentaciones contador de dosis. No contiene aditivos, inhalándose el fármaco puro por lo que el paciente no percibe la inhalación del mismo (Fig. 6).

Al estar el fármaco en un depósito, es más sensible a la humedad, pudiendo apelmazarse la medicación.

Técnica: ponerse de pie o sentado, desenroscar y retirar la capucha que cubre el inhalador, girar la base, primero hacia la dcha. y a continuación hacia la izqda. Tras dicho movimiento, se oirá un "clic", espirar profundamente, manteniendo el inhalador alejado de la boca, la

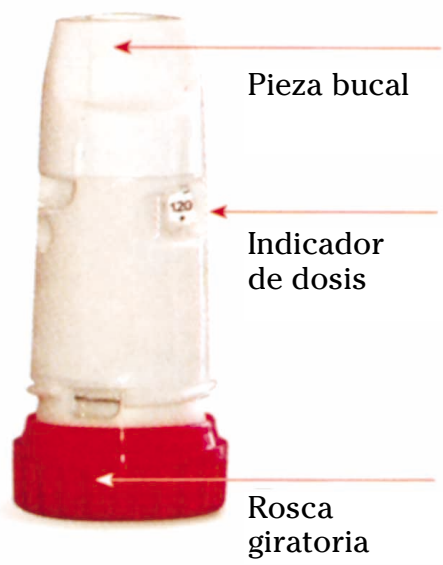

Figura 6. Sistema Turbuhaler. cabeza debe estar ligeramente inclinada hacia atrás, colocar la boquilla entre los dientes y cerrar los labios sobre la misma, inspirar profunda y sostenidamente, mantener la inspiración durante 10 segundos, espirar lentamente por la nariz, esperar un mínimo de 30 segundos si se debe repetir una segunda dosis del mismo u otro medicamento.

Mantenimiento: guardar en lugar seco y limpiar la boquilla con frecuencia.

Errores: girar la base sólo hacia la dcha., usar una vez agotadas las dosis, guardar en lugar húmedo, no expulsar el aire previo a la inhalación, espirar sobre el dispositivo, postura incorrecta (cabezatórax) y inspiración lenta.

\section{EDUCACIÓN SANITARIA}

La experiencia nos demuestra que la educación al paciente asmático es uno de los pilares fundamentales para el control del asma. Implica comprometer al paciente en el manejo de su propia enfermedad. Debemos procurar que el control del asma sea una responsabilidad compartida entre el paciente y el profesional de la salud. Para ello es fundamental conseguir una buena relación entre ambos, adiestrando al paciente en el conocimiento de síntomas y signos indicativos de su enfermedad para que de esta manera el propio paciente pueda tomar las medidas pertinentes. Además en pacientes con un asma severo debemos hacer el esfuerzo de enseñarles el manejo del medidor de pico de flujo para facilitarles el reconocimiento de su empeoramiento.

La educación es un proceso progresivo y continuado. No se debe sobrecargar a los pacientes con un exceso de información de una sola vez, y el lenguaje utilizado debe ser sencillo y directo con mensajes claros que se repitan constantemente.

Al paciente además de adiestrarle en el manejo de los diferentes inhaladores y su mantenimiento, se le debe de instruir en los siguientes aspectos:

- Después de cada inhalación de medicamento, enjuagar la boca y hacer gárgaras con agua, especialmente con los corti- 
coides, para prevenir la candidiasis orofaríngea.

- Si se está tomando simultáneamente un corticoide y un broncodilatador, utilizar primero el broncodilatador y esperar 10-20 minutos para aplicarse el corticoide.

- Es conveniente revisar periódicamente las técnicas de inhalación por parte del personal de enfermería para comprobar que se aplica la medicación correctamente o corregir posibles fallos.

\section{BIBLIOGRAFÍA}

1. Naberán KX, Calvo E, Garcia L, Hernández MF et al. Factores de riesgo. Manejo del asma en Atención Primaria. Recomendaciones SemFYC. Edide, Barcelona 1996; 13-18.

2. Caffarelli C, Cavagni G, Giordano S, Terzi V, PERRONE F. Reduced pulmonary function in multiple food-induced, exercise-related episodies of anaphylaxis. J Allergy Clin Immunol 1996; 98: 762-765.

3. Pauls JD, Cross D. Food-dependent exerciseinduced anaphylaxis to corn. J Allergy Clin Immunol 1998; 101: 853-854.

4. OWEns-Harrinson G, Grimm R, Gray D, Harrison O. Evaluation of education by a pharmacist to hospitalized patients who use metereddose inhalers. Hosp Pharm 1996; 31: 677-681.

5. Detullio PL, CoRson ME. Effect of pharmacist counseling on ambulatory use of aerosolized. Am J Hosp Pharm 1987; 44 1802-1806.

6. Rebuck D, Dzyngel B, Khan K, Kesten RN, CHAPMAN KR. The effect of structured versus conventional inhaler education in medical housestaff. J Asthma 1996; 33: 385-393.

7. United Nations Environment Programme. Report of the technology and economic assessment panel: Montreal protocol on substances that deplete the ozone layer. Nairobi, Kenia: United Nations environment Programme, Ozone Secretariat, 1995; 89-99.

8. Fisher DA, HALEs CH, WANG WC et al. Model calculations of the relative effects of CFCs and their replacements on global warning. Nature 1990; 344: 513-516.

9. Guidelines for the diagnosis and management of asthma. Expert panel report 2. NIH 1997.

10. BARLEY C. Inhalation devices and aerosol therapy. On Continuing Practice 1989; 16: 30 39

11. Clark DJ. Lipworth BJ. Effect of multiple actuations, delayed inhalation and antistatic treatment on the lung biovailability of salbutamol via a spacer device. Thorax 1996 51: 981-984.

12. Barry PW, O`CAllaghan C. Multiple actuations of salbutamol MDI into a spacer device reduce the amount of grug recovered in the respirable range. Eur Respir J 1994; 7 : 1707-1709.

13. O`Donohue WJ, Chair FCCP. National Association for Medical Direction of Respiratory Care Consensus Group. Guidelines for the use of nebulizers in the home and at domiciliary sites. Report of a consensus conference. Chest 1996; 109: 814820

14. Mandelberg A, Chen E, Noviski N, Priel IE. Nebulized wet aerosol treatment in emergency department-is it essential? Comparison with spacer device for metereddose inhaler. Chest 1997;112: 1501-1505.

15. BARRY PW, O`CALLAGHAN C. Inhalational drug delivery from seven different spacer devices. Thorax 1996; 51: 835-840.

16. Plaza V, Casan P, De Diego A, Duce F, Galdiz YB, LÓPEZ-VINA A et al. Recomendaciones para la utilización de fármacos inhalados. Arch Bronconeumol 1996; 32: 8-9.

17. Burnell PKP, Small T, DoIg $S$, Johal B, Jenkins R, GiBson GJ et al. Exvivo product performance of Diskus ${ }^{\mathrm{TM}}$ and Turbuhaler ${ }^{\mathrm{TM}}$ inhalers using inhalation profiles from patients with severe chronic obstructive pulmonary disease. Respir Med 2001; 95: 324-330.

18. HILL LS. Inhaler selection in asthma choosing the right device. Horizons January $1993 ; 29-32$. 